\title{
Pseudo-Groups, Moving Frames, and Differential Invariants
}

Peter J. Olver ${ }^{\dagger}$

School of Mathematics

University of Minnesota

Minneapolis, MN 55455

olver@math.umn.edu

http://www. math.umn. edu/ olver

\author{
Juha Pohjanpelto \\ Department of Mathematics \\ Oregon State University \\ Corvallis, OR 97331 \\ juha@math. oregonstate.edu \\ http://oregonstate.edu/ pohjanpp
}

\begin{abstract}
.
We survey recent developments in the method of moving frames for infinite-dimensional Lie pseudo-groups. These include a new, direct approach to the construction of invariant Maurer-Cartan forms and the Cartan structure equations for pseudo-groups, and new algorithms, based on constructive commutative algebra, for establishing the structure of their differential invariant algebras.
\end{abstract}

\section{Introduction.}

Lie pseudo-groups are the infinite-dimensional counterparts of local Lie groups of transformations. In Lie's day, abstract Lie groups were as yet unknown, and, as a result, no significant distinction was drawn between finite-dimensional and infinite-dimensional theory. However, since then the two subjects have traveled along radically different paths. The finite-dimensional theory has been rigorously formalized, and is a well-established and widely used mathematical tool. In contrast, the theory of infinite-dimensional pseudogroups remains surprisingly primitive in its current overall state of development. Since there is still no generally accepted abstract objects to play the role of infinite-dimensional Lie groups, Lie pseudo-groups only arise through their concrete action on a space. This makes the classification problems and analytical foundations of the subject thorny, particularly in the intransitive situation. We refer the reader to the original papers of Lie, Medolaghi, Tresse and Vessiot, $[\mathbf{3 6}, \mathbf{4 6}, \mathbf{6 9}, \mathbf{7 1}]$, for the classical theory of pseudo-groups,

$\dagger$ Supported in part by NSF Grant DMS 05-05293.

‡ Supported in part by NSF Grants DMS 04-53304 and OCE 06-21134.

January 26, 2007 
to Cartan, [12], for their reformulation in terms of exterior differential systems, and $[19,28,29,34,35,38,37,62,63,67,68]$ for a variety of modern approaches. Various nonconstructive approaches to the classification of differential invariants of Lie pseudogroups are studied in $[\mathbf{3 2}, \mathbf{3 3}, \mathbf{4 9}, \mathbf{6 9}]$.

Lie pseudo-groups appear in many fundamental physical and geometrical contexts, including gauge symmetries, [5], Hamiltonian mechanics and symplectic and Poisson geometry, [51], conformal geometry of surfaces and conformal field theory, $[\mathbf{1 8}, \mathbf{2 0}]$, the geometry of real hypersurfaces, [15], symmetry groups of both linear and nonlinear partial differential equations, such as the Navier-Stokes and Kadomtsev-Petviashvili (KP) equations appearing in fluid and plasma mechanics, $[\mathbf{4}, \mathbf{1 7}, \mathbf{5 1}]$, Vessiot's group splitting method

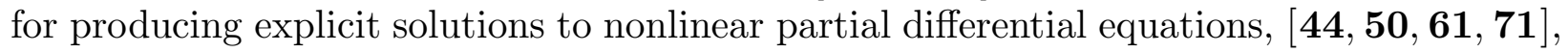
mathematical morphology and computer vision, $[\mathbf{6 6}, \mathbf{7 2}]$, and geometric numerical integration, $[45]$. Pseudogroups also appear as foliation-preserving groups of transformations, with the associated characteristic classes defined by certain invariant forms, cf. [23]. Also, keep in mind that all (sufficiently regular) local Lie group actions can be regarded as Lie pseudo-groups.

In a series of collaborative papers, starting with $[\mathbf{2 1}, \mathbf{2 2}]$, the first author has successfully reformulated the classical theory of moving frames, $[\mathbf{1 0}, \mathbf{2 4}]$, in a general, algorithmic, and equivariant framework that can be readily applied to a wide range of finite-dimensional Lie group actions. Applications have included complete classifications of differential invariants and their syzygies, [56], equivalence and symmetry properties of submanifolds, rigidity theorems, invariant signatures in computer vision, $[\mathbf{2}, \mathbf{6}, \mathbf{9}, \mathbf{5 4}]$, joint invariants and joint differential invariants, $[\mathbf{7}, \mathbf{5 4}]$, rational and algebraic invariants of algebraic group actions, $[\mathbf{2 6}, \mathbf{2 7}]$, invariant numerical algorithms, $[\mathbf{3 0}, \mathbf{5 5}, \mathbf{7 2}]$, classical invariant theory, $[\mathbf{3}, \mathbf{5 3}]$, Poisson geometry and solitons, $[\mathbf{4 1}, \mathbf{4 2}, \mathbf{4 3}]$, and the calculus of variations, $[\mathbf{3 1}]$. New applications of these methods to computation of symmetry groups and classification of

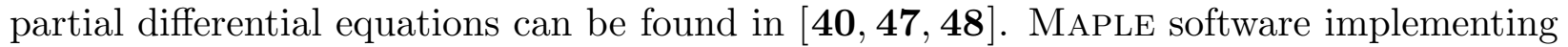
the moving frame algorithms, written by E. Hubert, can be found at [25]

Our main goal in this contribution is to survey the extension of the moving frame theory to general Lie pseudo-groups recently put forth by the authors in $[\mathbf{5 7}, \mathbf{5 8}, \mathbf{5 9}, \mathbf{6 0}]$, and in $[\mathbf{1 3}, \mathbf{1 4}]$ in collaboration with J. Cheh. Following $[\mathbf{3 1}]$, we develop the theory in the context of two different variational bicomplexes - the first over the infinite jet bundle $\mathcal{D}^{(\infty)} \subset \mathrm{J}^{\infty}(M, M)$ of local diffeomorphisms of $M$, and the second over the infinite jet bundle $\mathrm{J}^{\infty}(M, p)$ of $p$-dimensional submanifolds $N \subset M,[\mathbf{1}, \mathbf{3 1}, \mathbf{7 0}]$. The interplay between these two bicomplexes underlies our moving frame constructions. Importantly, the invariant contact forms on the diffeomorphism jet bundle $\mathcal{D}^{(\infty)}$ will play the role of Maurer-Cartan forms for the diffeomorphism pseudo-group. This identification enables us to explicitly formulate the diffeomorphism structure equations in power series form. Restricting the diffeomorphism-invariant forms to the pseudo-group subbundle $\mathcal{G}^{(\infty)} \subset \mathcal{D}^{(\infty)}$ yields a complete system of Maurer-Cartan forms for the pseudo-group. The remarkable fact is that the Maurer-Cartan forms satisfy an "invariantized" version of the linear infinitesimal determining equations for the pseudo-group, and, as a result, we can immediately produce an explicit form of the pseudo-group structure equations. Application of these results to the design of a practical computational algorithm for directly determining 
the structure of symmetry (pseudo-)groups of partial differential equations can be found in $[4,13,14,48]$.

Assuming freeness of the prolonged pseudo-group action at sufficiently high order, the explicit construction of the moving frame is founded on the Cartan normalization procedure associated with a choice of local cross-section to the pseudo-group orbits in $\mathrm{J}^{\infty}(M, p)$. The moving frame induces an invariantization process that projects general differential functions and differential forms on $\mathrm{J}^{\infty}(M, p)$ to invariant counterparts. In particular, invariantization of the standard jet coordinates results in a complete local system of normalized differential invariants, while invariantization of the horizontal and contact one-forms yields an invariant coframe. The corresponding dual invariant total derivative operators will map invariants to invariants of higher order. The structure of the algebra of differential invariants, including the specification of a finite generating set of differential invariants along with their syzygies or differential relations, will then follow from the recurrence formulae that relate the differentiated and normalized differential invariants. Remarkably, this final step requires only linear algebra and differentiation based on the infinitesimal determining equations of the pseudo-group action, and not the explicit formulae for either the differential invariants, the invariant differential operators, or the moving frame. Except possibly for some low order complications, the underlying structure of the differential invariant algebra is then entirely governed by two commutative algebraic modules: the symbol module of the infinitesimal determining system of the pseudo-group and a new module, named the "prolonged symbol module", containing the symbols of the prolonged infinitesimal generators.

The paper begins with a discussion of the most basic example - the diffeomorphism pseudo-group of a manifold. The usual variational bicomplex structure on the diffeomorphism jets is employed to construct the Maurer-Cartan forms as invariant contact forms, and write out the complete system of structure equations. Section 3 shows how the structure equations of a Lie pseudo-group are obtained by restricting the diffeomorphism structure equations to the solution space to the infinitesimal determining equations. In section 4 , we develop the moving frame constructions for the prolonged action on submanifold jets, and explain how to determine a complete system of differential invariants. In section 5, we explicitly derive the recurrence formulae for the differentiated invariants, demonstrating, in particular, that the differential invariants of any transitive pseudo-group form a non-commutative rational differential algebra. Finally, in section 6 we present a constructive version of the Basis Theorem that provides a finite system of generating differential invariants for a large class of pseudo-group actions and the generators of their differential syzygies. Lack of space precludes us from including any serious examples, and, for this, we refer the reader to $[\mathbf{1 3}, \mathbf{1 4}, \mathbf{5 8}, \mathbf{5 9}, \mathbf{6 0}]$.

\section{The Diffeomorphism Pseudo-Group.}

Let $M$ be a smooth $m$-dimensional manifold. Let $\mathcal{D}=\mathcal{D}(M)$ denote the pseudogroup of all local diffeomorphisms $\varphi: M \rightarrow M$. For each $0 \leq n \leq \infty$, let $\mathcal{D}^{(n)}=$ $\mathcal{D}^{(n)}(M) \subset \mathrm{J}^{n}(M, M)$ denote the $n^{\text {th }}$ order diffeomorphism jet groupoid, [39], with source $\operatorname{map} \boldsymbol{\sigma}^{(n)}\left(\left.\mathrm{j}_{n} \varphi\right|_{z}\right)=z$ and target map $\boldsymbol{\tau}^{(n)}\left(\left.\mathrm{j}_{n} \varphi\right|_{z}\right)=\varphi(z)=Z$. The groupoid multiplication is induced by composition of diffeomorphisms. Following Cartan, [11, 12], we 
will consistently use lower case letters, $z, x, u, \ldots$ for the source coordinates and the corresponding upper case letters $Z, X, U, \ldots$ for the target coordinates of our diffeomorphisms $\varphi$. Given local coordinates $(z, Z)=\left(z^{1}, \ldots, z^{m}, Z^{1}, \ldots, Z^{m}\right)$ on an open subset of $M \times M$, the induced local coordinates of $g^{(n)}=\left.\mathrm{j}_{n} \varphi\right|_{z} \in \mathcal{D}^{(n)}$ are denoted $\left(z, Z^{(n)}\right)$, where the components $Z_{B}^{a}$ of $Z^{(n)}$, for $a=1, \ldots, m, \# B \leq n$, represent the partial derivatives $\partial^{B} \varphi^{a} / \partial z^{B}$ of $\varphi$ at the source point $z=\boldsymbol{\sigma}^{(n)}\left(g^{(n)}\right)$.

Since $\mathcal{D}^{(\infty)} \subset \mathrm{J}^{\infty}(M, M)$, the inherited variational bicomplex structure, $[\mathbf{1}, \mathbf{7 0}]$, provides a natural splitting of the cotangent bundle $T^{*} \mathcal{D}^{(\infty)}$ into horizontal and vertical (contact) components, $[\mathbf{1}, \mathbf{5 2}]$, and we use $d=d_{M}+d_{G}$ to denote the induced splitting of the differential. In terms of local coordinates $g^{(\infty)}=\left(z, Z^{(\infty)}\right)$, the horizontal subbundle of $T^{*} \mathcal{D}^{(\infty)}$ is spanned by the one-forms $d z^{a}=d_{M} z^{a}, a=1, \ldots, m$, while the vertical subbundle is spanned by the basic contact forms

$$
\Upsilon_{B}^{a}=d_{G} Z_{B}^{a}=d Z_{B}^{a}-\sum_{c=1}^{m} Z_{B, c}^{a} d z^{c}, \quad a=1, \ldots, m, \quad \# B \geq 0 .
$$

Composition of local diffeomorphisms induces an action of $\psi \in \mathcal{D}$ by right multiplication on diffeomorphism jets: $\mathrm{R}_{\psi}\left(\left.\mathrm{j}_{n} \varphi\right|_{z}\right)=\left.\mathrm{j}_{n}\left(\varphi \circ \psi^{-1}\right)\right|_{\psi(z)}$. A differential form $\mu$ on $\mathcal{D}^{(n)}$ is right-invariant if $\mathrm{R}_{\psi}^{*} \mu=\mu$, where defined, for every $\psi \in \mathcal{D}$. Since the splitting of forms on $\mathcal{D}^{(\infty)}$ is invariant under this action, if $\mu$ is any right-invariant differential form, so are $d_{M} \mu$ and $d_{G} \mu$. The target coordinate functions $Z^{a}: \mathcal{D}^{(0)} \rightarrow \mathbb{R}$ are obviously right-invariant, and hence their horizontal differentials

$$
\sigma^{a}=d_{M} Z^{a}=\sum_{b=1}^{m} Z_{b}^{a} d z^{b}, \quad a=1, \ldots, m
$$

form an invariant horizontal coframe, while their vertical differentials

$$
\mu^{a}=d_{G} Z^{a}=\Upsilon^{a}=d Z^{a}-\sum_{b=1}^{m} Z_{b}^{a} d z^{b}, \quad a=1, \ldots, m
$$

are the zero ${ }^{\text {th }}$ order invariant contact forms. Let $\mathbb{D}_{Z^{1}}, \ldots, \mathbb{D}_{Z^{m}}$ be the total derivative operators dual to the horizontal forms $(2.2)$, so that

$$
d_{M} F=\sum_{a=1}^{m} \mathbb{D}_{z^{a}} F d z^{a} \quad \text { for any } \quad F: \mathcal{D}^{(\infty)} \rightarrow \mathbb{R}
$$

Then the higher-order invariant contact forms are obtained by successively Lie differentiating the invariant contact forms (2.3):

$$
\mu_{B}^{a}=\mathbb{D}_{Z}^{B} \mu^{a}=\mathbb{D}_{Z}^{B} \Upsilon^{a}, \quad \text { where } \quad \mathbb{D}_{Z}^{B}=\mathbb{D}_{Z^{b_{1}}} \cdots \mathbb{D}_{Z^{b_{k}}}, \quad \begin{array}{ll}
a & =1, \ldots, m, \\
& k=\# B \geq 0 .
\end{array}
$$

As explained in $[\mathbf{5 8}]$, the right-invariant contact forms $\mu^{(\infty)}=\left(\ldots \mu_{B}^{a} \ldots\right)$ are to be viewed as the Maurer-Cartan forms for the diffeomorphism pseudo-group. 
The next step in our program is to establish the structure equations for the diffeomorphism groupoid $\mathcal{D}^{(\infty)}$. Let $\mu \llbracket H \rrbracket$ denote the column vector whose components are the invariant contact form-valued formal power series

$$
\mu^{a} \llbracket H \rrbracket=\sum_{\# B \geq 0} \frac{1}{B !} \mu_{B}^{a} H^{B}, \quad a=1, \ldots, m,
$$

depending on the formal parameters $H=\left(H^{1}, \ldots, H^{m}\right)$. Further, let $d Z=\mu \llbracket 0 \rrbracket+\sigma$ denote column vectors of one-forms whose entries are $d Z^{a}=\mu^{a}+\sigma^{a}$ for $a=1, \ldots, m$.

Theorem 2.1. The complete structure equations for the diffeomorphism pseudogroup are obtained by equating coefficients in the power series identity

$$
d \mu \llbracket H \rrbracket=\nabla_{H} \mu \llbracket H \rrbracket \wedge(\mu \llbracket H \rrbracket-d Z), \quad d \sigma=-d \mu \llbracket 0 \rrbracket=\nabla_{H} \mu \llbracket 0 \rrbracket \wedge \sigma,
$$

where $\nabla_{H} \mu \llbracket H \rrbracket=\left(\frac{\partial \mu^{a}}{\partial H^{b}} \llbracket H \rrbracket\right)$ denotes the $m \times m$ formal power series Jacobian matrix.

The key to analyzing pseudo-group actions is to work infinitesimally, using the generating Lie algebra ${ }^{\dagger}$ of vector fields. Let $\mathcal{X}(M)$ denote the space of locally defined vector fields on $M$, which we write in local coordinates as

$$
\mathbf{v}=\sum_{a=1}^{m} \zeta^{a}(z) \frac{\partial}{\partial z^{a}}
$$

Let $\mathrm{J}^{n} T M$, for $0 \leq n \leq \infty$, denote the tangent $n$-jet bundle. Local coordinates on $\mathrm{J}^{n} T M$

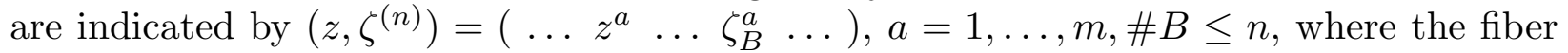
coordinate $\zeta_{B}^{a}$ represents the partial derivative $\partial^{B} \zeta^{a} / \partial z^{B}$.

\section{Lie Pseudo-Groups.}

The literature contains several variants of the precise technical definition of a Lie pseudo-group. Ours is:

Definition 3.1. A sub-pseudo-group $\mathcal{G} \subset \mathcal{D}$ will be called a Lie pseudo-group if there exists $n_{0} \geq 1$ such that for all finite $n \geq n_{0}$ :

(a) the corresponding sub-groupoid $\mathcal{G}^{(n)} \subset \mathcal{D}^{(n)}$ forms a smooth, embedded subbundle,

(b) every smooth local solution $Z=\varphi(z)$ to the determining system $\mathcal{G}^{(n)}$ belongs to $\mathcal{G}$,

(c) $\mathcal{G}^{(n)}=\operatorname{pr}^{\left(n-n_{0}\right)} \mathcal{G}^{\left(n_{0}\right)}$ is obtained by prolongation.

$\dagger$ Here, we are using the term "Lie algebra" imprecisely, since, technically, the vector fields may only be locally defined, and so their Lie brackets only make sense on their common domains of definition. 
The minimal value of $n_{0}$ is called the order of the pseudo-group.

Thus on account of conditions (a) and (c), for $n \geq n_{0}$, the pseudo-group jet subgroupoid $\mathcal{G}^{(n)} \subset \mathcal{D}^{(n)}$ is defined in local coordinates by a formally integrable system of $n^{\text {th }}$ order nonlinear partial differential equations

$$
F^{(n)}\left(z, Z^{(n)}\right)=0
$$

known as the determining equations for the pseudo-group. Condition (b) says that the local solutions $Z=\varphi(z)$ to the determining equations are precisely the pseudo-group transformations.

Let $\mathfrak{g} \subset \mathcal{X}$ denote the Lie algebra of infinitesimal generators of the pseudo-group, i.e., the set of locally defined vector fields $(2.8)$ whose flows belong to $\mathcal{G}$. In local coordinates, we can view $\mathrm{J}^{n} \mathfrak{g} \subset \mathrm{J}^{n} T M$ as defining a formally integrable linear system of partial differential equations

$$
L^{(n)}\left(z, \zeta^{(n)}\right)=0
$$

for the vector field coefficients (2.8), called the linearized or infinitesimal determining equations for the pseudo-group. They can be obtained by linearizing the $n^{\text {th }}$ order determining equations (3.1) at the identity jet. If $\mathcal{G}$ is the symmetry group of a system of differential equations, then the linearized determining equations (3.2) are (the involutive completion of) the usual determining equations for its infinitesimal generators obtained via Lie's algorithm, [51].

As with finite-dimensional Lie groups, the structure of a pseudo-group is described by its invariant Maurer-Cartan forms. A complete system of right-invariant one-forms on $\mathcal{G}^{(\infty)} \subset \mathcal{D}^{(\infty)}$ is obtained by restricting (or pulling back) the Maurer-Cartan forms (2.2-5). For simplicity, we continue to denote these forms by $\sigma^{a}, \mu_{B}^{a}$. The restricted Maurer-Cartan forms are, of course, no longer linearly independent, but are subject to certain constraints prescribed by the pseudo-group. Remarkably, these constraints can be explicitly characterized by an invariant version of the linearized determining equations (3.2), which is formally obtained by replacing the source coordinates $z^{a}$ by the corresponding target coordinates $Z^{a}$ and the vector field jet coordinates $\zeta_{B}^{a}$ by the corresponding Maurer-Cartan form $\mu_{B}^{a}$.

Theorem 3.2. The linear system

$$
L^{(n)}\left(Z, \mu^{(n)}\right)=0
$$

serves to define the complete set of dependencies among the right-invariant Maurer-Cartan forms $\mu^{(n)}$ on $\mathcal{G}^{(n)}$. Therefore, the structure equations for the pseudo-group $\mathcal{G}$ are obtained by restriction of the diffeomorphism structure equations (2.7) to the kernel of the linearized involutive system (3.3).

In this way, we effectively and efficiently bypass Cartan's more complicated prolongation procedure, $[\mathbf{8}, \mathbf{1 2}]$, for accessing the pseudo-group structure equations. Examples of this procedure can be found in $[\mathbf{1 3}, \mathbf{5 8}]$; see also $[\mathbf{4 8}]$ for a comparison with other approaches. 
Example 3.3. Let us consider the pseudo-group

$$
X=f(x), \quad Y=e(x, y) \equiv f^{\prime}(x) y+g(x), \quad U=u+\frac{e_{x}(x, y)}{f^{\prime}(x)}=u+\frac{f^{\prime \prime}(x) y+g^{\prime}(x)}{f^{\prime}(x)},
$$

acting on $M=\mathbb{R}^{3}$, with local coordaintes $(x, y, u)$. Here $f(x) \in \mathcal{D}(\mathbb{R})$, while $g(x) \in \mathrm{C}^{\infty}(\mathbb{R})$. The determining equations are the first order involutive system

$$
X_{y}=X_{u}=0, \quad Y_{y}=X_{x} \neq 0, \quad Y_{u}=0, \quad Y_{x}=(U-u) X_{x}, \quad U_{u}=1 .
$$

The infinitesimal generators of the pseudo-group have the form

$$
\mathbf{v}=\xi \frac{\partial}{\partial x}+\eta \frac{\partial}{\partial y}+\varphi \frac{\partial}{\partial u}=a(x) \frac{\partial}{\partial x}+\left[a^{\prime}(x) y+b(x)\right] \frac{\partial}{\partial y}+\left[a^{\prime \prime}(x) y+b^{\prime}(x)\right] \frac{\partial}{\partial u},
$$

where $a(x), b(x)$ are arbitrary smooth functions. The infinitesimal generators (3.6) form the general solution to the first order involutive infinitesimal determining system

$$
\xi_{x}=\eta_{y}, \quad \xi_{y}=\xi_{u}=\eta_{u}=\varphi_{u}=0, \quad \eta_{x}=\varphi,
$$

obtained by linearizing (3.5) at the identity.

The Maurer-Cartan forms are obtained by repeatedly differentiating $\mu=d_{G} X, \widetilde{\mu}=$ $d_{G} Y$ and $\nu=d_{G} U$, so that $\mu_{j, k, l}=\mathbb{D}_{X}^{j} \mathbb{D}_{Y}^{k} \mathbb{D}_{U}^{l} \mu$, etc. According to Theorem 3.2, they are subject to the linear relations

$$
\mu_{X}=\widetilde{\mu}_{Y}, \quad \mu_{Y}=\mu_{U}=\widetilde{\mu}_{U}=\nu_{U}=0, \quad \widetilde{\mu}_{X}=\nu,
$$

along with their "differential" consequences. Writing out (2.7), we are led to the following structure equations

$$
\begin{aligned}
d \mu_{n} & =\sigma \wedge \mu_{n+1}-\sum_{j=1}^{[(n+1) / 2]} \frac{n-2 j+1}{n+1}\left(\begin{array}{c}
n+1 \\
j
\end{array}\right) \mu_{j} \wedge \mu_{n+1-j}, \\
d \widetilde{\mu}_{n} & =\sigma \wedge \widetilde{\mu}_{n+1}+\widetilde{\sigma} \wedge \mu_{n+1}-\sum_{j=0}^{n-1} \frac{n-2 j-1}{n+1}\left(\begin{array}{c}
n+1 \\
j+1
\end{array}\right) \widetilde{\mu}_{j+1} \wedge \mu_{n-j}, \\
d \sigma & =-d \mu=-\sigma \wedge \mu_{X}, \\
d \widetilde{\sigma} & =-d \widetilde{\mu}=-\sigma \wedge \widetilde{\mu}_{X}-\widetilde{\sigma} \wedge \mu_{X}, \\
d \tau & =-d \nu=-d \widetilde{\mu}_{X}=-\sigma \wedge \widetilde{\mu}_{X X}-\widetilde{\sigma} \wedge \mu_{X X},
\end{aligned}
$$

in which $\sigma=d_{M} X, \widetilde{\sigma}=d_{M} Y, \tau=d_{M} U$, and $\mu_{n}=\mu_{n, 0,0}, \widetilde{\mu}_{n}=\widetilde{\mu}_{n, 0,0}$, for $n=0,1,2, \ldots$, form a basis for the Maurer-Cartan forms of the pseudo-group. See [58] for full details.

\section{Pseudo-Group Actions on Extended Jet Bundles.}

Our primary focus is to study the induced action of pseudo-groups on submanifolds. For $0 \leq n \leq \infty$, let $\mathrm{J}^{n}=\mathrm{J}^{n}(M, p)$ denote the $n^{\text {th }}$ order (extended) jet bundle consisting of 
equivalence classes of $p$-dimensional submanifolds $S \subset M$ under the equivalence relation of $n^{\text {th }}$ order contact, cf. [52]. We employ the standard local coordinates

$$
z^{(n)}=\left(x, u^{(n)}\right)=\left(\ldots x^{i} \ldots u_{J}^{\alpha} \ldots\right)
$$

on $\mathrm{J}^{n}$ induced by a splitting of the local coordinates $z=(x, u)=\left(x^{1}, \ldots, x^{p}, u^{1}, \ldots, u^{q}\right)$ on $M$ into $p$ independent and $q=m-p$ dependent variables, $[\mathbf{5 1}, \mathbf{5 2}]$. The choice of independent and dependent variables induces the variational bicomplex structure on $\mathrm{J}^{\infty}$, $[\mathbf{1}, \mathbf{7 0}]$. The basis horizontal forms are the differentials $d x^{1}, \ldots, d x^{p}$ of the independent variables, while the basis contact forms are denoted by

$$
\theta_{J}^{\alpha}=d u_{J}^{\alpha}-\sum_{i=1}^{p} u_{J, i}^{\alpha} d x^{i}, \quad \alpha=1, \ldots, q, \quad \# J \geq 0 .
$$

This decomposition splits the differential $d=d_{H}+d_{V}$ on $\mathrm{J}^{\infty}$ into horizontal and vertical (or contact) components, and endows the space of differential forms with the structure of a variational bicomplex, $[\mathbf{1}, \mathbf{3 1}, \mathbf{7 0}]$.

Local diffeomorphisms $\varphi \in \mathcal{D}$ preserve the contact equivalence relation between submanifolds, and thus induce an action on the jet bundle $\mathrm{J}^{n}=\mathrm{J}^{n}(M, p)$, known as the $n^{\text {th }}$ prolonged action, which, by the chain rule, factors through the diffeomorphism jet groupoid $\mathcal{D}^{(n)}$. Let $\mathcal{H}^{(n)}$ denote the groupoid obtained by pulling back the pseudo-group jet groupoid $\mathcal{G}^{(n)} \rightarrow M$ via the projection $\tilde{\pi}_{0}^{n}: \mathrm{J}^{n} \rightarrow M$. Local coordinates on $\mathcal{H}^{(n)}$ are written $\left(x, u^{(n)}, g^{(n)}\right)$, where $\left(x, u^{(n)}\right)$ are the coordinates and $\mathrm{J}^{n}(M, p)$, while the fiber coordinates $g^{(n)}$ serve to parametrize the pseudo-group jets.

Definition 4.1. A moving frame $\rho^{(n)}$ of order $n$ is a $\mathcal{G}^{(n)}$ equivariant local section of the bundle $\mathcal{H}^{(n)} \rightarrow \mathrm{J}^{n}$.

Thus, in local coordinates, the moving frame section has the form

$$
\rho^{(n)}\left(x, u^{(n)}\right)=\left(x, u^{(n)}, \gamma^{(n)}\left(x, u^{(n)}\right)\right), \quad \text { where } \quad g^{(n)}=\gamma^{(n)}\left(x, u^{(n)}\right)
$$

defines a right equivariant map to the pseudo-group jets. A moving frame $\rho^{(k)}: \mathrm{J}^{k} \rightarrow$ $\mathcal{H}^{(k)}$ of order $k>n$ is compatible with $\rho^{(n)}$ provided $\widehat{\pi}_{n}^{k} \circ \rho^{(k)}=\rho^{(n)} \circ \widetilde{\pi}_{n}^{k}$ where defined, $\widehat{\pi}_{n}^{k}: \mathcal{H}^{(k)} \rightarrow \mathcal{H}^{(n)}$ and $\widetilde{\pi}_{n}^{k}: \mathrm{J}^{k} \rightarrow \mathrm{J}^{n}$ denoting the evident projections. A complete moving frame is provided by a mutually compatible collection of moving frames of all orders $k \geq n$.

As in the finite-dimensional construction, [22], the (local) existence of a moving frame requires that the prolonged pseudo-group action be free and regular.

Definition 4.2. The pseudo-group $\mathcal{G}$ acts freely at $z^{(n)} \in \mathrm{J}^{n}$ if its isotropy subgroup is trivial, $\mathcal{G}_{z^{(n)}}^{(n)}=\left\{\mathbf{g}^{(n)} \in \mathcal{G}^{(n)} \mid \mathbf{g}^{(n)} \cdot z^{(n)}=z^{(n)}\right\}=\left\{\mathbb{1}_{z}^{(n)}\right\}$, and locally freely if $\mathcal{G}_{z^{(n)}}^{(n)}$ is discrete.

Warning: According to the standard definition, [22], any (locally) free action of a finite-dimensional Lie group satisfies the (local) freeness condition of Definition 4.2, but not necessarily conversely. 
The pseudo-group acts locally freely at $z^{(n)}$ if and only if the prolonged pseudo-group orbit through $z^{(n)}$ has dimension $r_{n}=\left.\operatorname{dim} \mathcal{G}^{(n)}\right|_{z}$. Thus, freeness of the pseudo-group at order $n$ requires, at the very least, that

$$
r_{n}=\left.\operatorname{dim} \mathcal{G}^{(n)}\right|_{z} \leq \operatorname{dim} \mathrm{J}^{n}=p+(m-p)\left(\begin{array}{c}
p+n \\
p
\end{array}\right) .
$$

Freeness thus provides an alternative and simpler means of quantifying the Spencer cohomological growth conditions imposed on the pseudo-group in $[\mathbf{3 2}, 33]$. Pseudo-groups having too large a fiber dimension $r_{n}$ will, typically, act transitively on (a dense open subset of) $\mathrm{J}^{n}$, and thus possess no non-constant differential invariants. A key result of [60], generalizing the finite-dimensional case, is the persistence of local freeness.

Theorem 4.3. Let $\mathcal{G}$ be a Lie pseudo-group acting on an $m$-dimensional manifold $M$. If $\mathcal{G}$ acts locally freely at $z^{(n)} \in \mathrm{J}^{n}$ for some $n>0$, then it acts locally freely at any $z^{(k)} \in \mathrm{J}^{k}$ with $\widetilde{\pi}_{n}^{k}\left(z^{(k)}\right)=z^{(n)}$, for $k \geq n$.

As in the finite-dimensional version, $[\mathbf{2 2}]$, moving frames are constructed through a normalization procedure based on a choice of cross-section to the pseudo-group orbits, i.e., a transverse submanifold of the complementary dimension.

Theorem 4.4. Suppose $\mathcal{G}^{(n)}$ acts freely on an open subset $\mathcal{V}^{n} \subset \mathrm{J}^{n}$, with its orbits forming a regular foliation. Let $K^{n} \subset \mathcal{V}^{n}$ be a (local) cross-section to the pseudo-group orbits. Given $z^{(n)} \in \mathcal{V}^{n}$, define $\rho^{(n)}\left(z^{(n)}\right) \in \mathcal{H}^{(n)}$ to be the unique pseudo-group jet such that $\widetilde{\boldsymbol{\sigma}}^{(n)}\left(\rho^{(n)}\left(z^{(n)}\right)\right)=z^{(n)}$ and $\widetilde{\boldsymbol{\tau}}^{(n)}\left(\rho^{(n)}\left(z^{(n)}\right)\right) \in K^{n}$ (when such exists). Then $\rho^{(n)}: \mathrm{J}^{n} \rightarrow \mathcal{H}^{(n)}$ is a moving frame for $\mathcal{G}$ defined on an open subset of $\mathcal{V}^{n}$ containing $K^{n}$.

Usually - and, to simplify the development, from here on - we select a coordinate cross-section of minimal order, defined by fixing the values of $r_{n}$ of the individual submanifold jet coordinates $\left(x, u^{(n)}\right)$. We write out the explicit formulae $\left(X, U^{(n)}\right)=$ $F^{(n)}\left(x, u^{(n)}, g^{(n)}\right)$ for the prolonged pseudo-group action in terms of a convenient system of group parameters $g^{(n)}=\left(g_{1}, \ldots, g_{r_{n}}\right)$. The $r_{n}$ components corresponding to our choice of cross-section variables serve to define the normalization equations

$$
F_{1}\left(x, u^{(n)}, g^{(n)}\right)=c_{1}, \quad \ldots \quad F_{r_{n}}\left(x, u^{(n)}, g^{(n)}\right)=c_{r_{n}},
$$

which, when solved for the group parameters $g^{(n)}=\gamma^{(n)}\left(x, u^{(n)}\right)$, produces the moving frame section (4.3).

With the moving frame in place, the general invariantization procedure introduced in [31] in the finite-dimensional case adapts straightforwardly. To compute the invariantization of a function, differential form, differential operator, etc., one writes out how it explicitly transforms under the pseudo-group, and then replaces the pseudo-group parameters by their moving frame expressions (4.3). Invariantization defines a morphism that projects the exterior algebra differential functions and forms onto the algebra of invariant differential functions and forms. In particular, invariantizing the coordinate functions on $\mathrm{J}^{\infty}$ leads to the normalized differential invariants

$$
H^{i}=\iota\left(x^{i}\right), \quad i=1, \ldots, p, \quad I_{J}^{\alpha}=\iota\left(u_{J}^{\alpha}\right), \quad \alpha=1, \ldots, q, \quad \# J \geq 0,
$$


collectively denoted by $\left(H, I^{(n)}\right)=\iota\left(x, u^{(n)}\right)$. The normalized differential invariants naturally split into two subspecies: those appearing in the normalization equations (4.5) will be constant, and are known as the phantom differential invariants. The remaining $s_{n}=\operatorname{dim} \mathrm{J}^{n}-r_{n}$ components, called the basic differential invariants, form a complete system of functionally independent differential invariants of order $\leq n$ for the prolonged pseudo-group action on submanifolds.

Secondly, invariantization of the basis horizontal one-forms leads to the invariant oneforms

$$
\varpi^{i}=\iota\left(d x^{i}\right)=\omega^{i}+\kappa^{i}, \quad i=1, \ldots, p,
$$

where $\omega^{i}, \kappa^{i}$ denote, respectively, the horizontal and vertical (contact) components. If the pseudo-group acts projectably, then the contact components vanish: $\kappa^{i}=0$. The horizontal forms $\omega^{1}, \ldots, \omega^{p}$ provide, in the language of $[\mathbf{5 2}]$, a contact-invariant coframe on $\mathrm{J}^{\infty}$. The dual invariant differential operators $\mathcal{D}_{1}, \ldots, \mathcal{D}_{p}$ are uniquely defined by the formula

$$
d F=\sum_{i=1}^{p} \mathcal{D}_{i} F \varpi^{i}+\cdots,
$$

valid for any differential function $F$, where the dots indicate contact components which are not needed here, but do play an important role in the study of invariant variational problems, cf. [31]. The invariant differential operators $\mathcal{D}_{i}$ map differential invariants to differential invariants. In general, they do not commute, but are subject to linear commutation relations of the form

$$
\left[\mathcal{D}_{i}, \mathcal{D}_{j}\right]=\sum_{k=1}^{p} Y_{i j}^{k} \mathcal{D}_{k}, \quad i, j=1, \ldots, p,
$$

where the coefficients $Y_{i j}^{k}$ are certain differential invariants. Finally, invariantizing the basis contact one-forms

$$
\vartheta_{K}^{\alpha}=\iota\left(\theta_{K}^{\alpha}\right), \quad \alpha=1, \ldots, q, \quad \# K \geq 0,
$$

provide a complete system of invariant contact one-forms. The invariant coframe serves to define the invariant variational complex for the pseudo-group, [31].

The Basis Theorem for differential invariants states that, assuming freeness of the sufficiently high order prolonged pseudo-group action, then locally, there exist a finite number of generating differential invariants $I_{1}, \ldots, I_{\ell}$, with the property that every differential invariant can be locally expressed as a function of the generating invariants and their invariant derivatives:

$$
\mathcal{D}_{J} I_{\kappa}=\mathcal{D}_{j_{1}} \mathcal{D}_{j_{2}} \cdots \mathcal{D}_{j_{k}} I_{\kappa}
$$

The differentiated invariants are not necessarily independent, but may be subject to certain functional relations or differential syzygies of the form

$$
H\left(\ldots \mathcal{D}_{J} I_{\kappa} \ldots\right) \equiv 0 .
$$

A consequence of our moving frame methods is a constructive algorithm for producing a (not necessarily minimal) system of generating differential invariants, as well as a proof that 
there are finitely many generating syzygies, meaning that any other syzygy is a differential consequence thereof.

Example 4.5. Consider the action of the pseudo-group (3.4) on surfaces $u=h(x, y)$. Under the pseudo-group transformations, the basis horizontal forms $d x, d y$ are mapped to the one-forms

$$
d_{H} X=f_{x} d x, \quad d_{H} Y=e_{x} d x+f_{x} d y .
$$

The prolonged pseudo-group transformations are found by applying the dual implicit differentiations

$$
\mathrm{D}_{X}=\frac{1}{f_{x}} \mathrm{D}_{x}-\frac{e_{x}}{f_{x}^{2}} \mathrm{D}_{y}, \quad \mathrm{D}_{Y}=\frac{1}{f_{x}} \mathrm{D}_{y},
$$

successively to $U=u+e_{x} / f_{x}$, so that

$$
\begin{aligned}
& U_{X}=\frac{u_{x}}{f_{x}}+\frac{e_{x x}-e_{x} u_{y}}{f_{x}^{2}}-2 \frac{f_{x x} e_{x}}{f_{x}^{3}}, \quad U_{Y}=\frac{u_{y}}{f_{x}}+\frac{f_{x x}}{f_{x}^{2}}, \\
& U_{X X}=\frac{u_{x x}}{f_{x}^{2}}+\frac{e_{x x x}-e_{x x} u_{y}-2 e_{x} u_{x y}-f_{x x} u_{x}}{f_{x}^{3}}+ \\
& +\frac{e_{x}^{2} u_{y y}+3 e_{x} f_{x x} u_{y}-4 e_{x x} f_{x x}-3 e_{x} f_{x x x}}{f_{x}^{4}}+8 \frac{e_{x} f_{x x}^{2}}{f_{x}^{5}}, \\
& U_{X Y}=\frac{u_{x y}}{f_{x}^{2}}+\frac{f_{x x x}-f_{x x} u_{y}-e_{x} u_{y y}}{f_{x}^{3}}-2 \frac{f_{x x}^{2}}{f_{x}^{4}}, \quad U_{Y Y}=\frac{u_{y y}}{f_{x}^{2}},
\end{aligned}
$$

and so on. In these formulae, the jet coordinates $f, f_{x}, f_{x x}, \ldots, e, e_{x}, e_{x x}, \ldots$ are to be regarded as the independent pseudo-group parameters. The pseudo-group cannot act freely on $\mathrm{J}^{1}$ since $r_{1}=\left.\operatorname{dim} \mathcal{G}^{(1)}\right|_{z}=6>\operatorname{dim} \mathrm{J}^{1}=5$. On the other hand, $r_{2}=\left.\operatorname{dim} \mathcal{G}^{(2)}\right|_{z}=$ $8=\operatorname{dim} \mathrm{J}^{2}$, and the action on $\mathrm{J}^{2}$ is, in fact, locally free and transitive on the sets $\mathcal{V}^{2}{ }_{+}=$ $\mathrm{J}^{2} \cap\left\{u_{y y}>0\right\}$ and $\mathcal{V}^{2}=\mathrm{J}^{2} \cap\left\{u_{y y}<0\right\}$. Moreover, as predicted by Theorem 4.3, $\mathcal{G}^{(n)}$ acts locally freely on the corresponding open subsets of $\mathrm{J}^{n}$ for any $n \geq 2$.

To construct the moving frame, we successively solve the following coordinate crosssection equations for the pseudo-group parameters:

$$
\begin{aligned}
X & =0, & & f=0, \\
Y & =0, & & e=0, \\
U & =0, & & e_{x}=-u f_{x}, \\
U_{Y} & =0, & & f_{x x}=-u_{y} f_{x}, \\
U_{X} & =0, & & e_{x x}=\left(u u_{y}-u_{x}\right) f_{x}, \\
U_{Y Y} & =1, & & f_{x}=\sqrt{u_{y y}}, \\
U_{X Y} & =0, & & f_{x x x}=-\sqrt{u_{y y}}\left(u_{x y}+u u_{y y}-u_{y}^{2}\right), \\
U_{X X} & =0, & & e_{x x x}=-\sqrt{u_{y y}}\left(u_{x x}-u u_{x y}-2 u^{2} u_{y y}-2 u_{x} u_{y}+u u_{y}^{2}\right) .
\end{aligned}
$$

At this stage, we can construct the first two fundamental differential invariants:

$$
J_{1}=\iota\left(u_{x y y}\right)=\frac{u_{x y y}+u u_{y y y}+2 u_{y} u_{y y}}{u_{y y}^{3 / 2}}, \quad J_{2}=\iota\left(u_{y y y}\right)=\frac{u_{y y y}}{u_{y y}^{3 / 2}} .
$$


Higher order differential invariants are found by continuing this procedure, or by employing the more powerful Taylor series method developed in [59]. Further, substituting the pseudo-group normalizations into (4.12) fixes the invariant horizontal coframe

$$
\omega^{1}=\iota(d x)=\sqrt{u_{y y}} d x, \quad \omega^{2}=\iota(d y)=\sqrt{u_{y y}}(d y-u d x) .
$$

The dual invariant total derivative operators are

$$
\mathcal{D}_{1}=\frac{1}{\sqrt{u_{y y}}}\left(\mathrm{D}_{x}+u \mathrm{D}_{y}\right), \quad \mathcal{D}_{2}=\frac{1}{\sqrt{u_{y y}}} \mathrm{D}_{y} .
$$

The higher-order differential invariants can be generated by successively applying these differential operators to the pair of basic differential invariants (4.14). The commutation relation is

$$
\left[\mathcal{D}_{1}, \mathcal{D}_{2}\right]=-\frac{1}{2} J_{2} \mathcal{D}_{1}+\frac{1}{2} J_{1} \mathcal{D}_{2}
$$

Finally, there is a single generating syzygy

$$
\mathcal{D}_{1} J_{2}-\mathcal{D}_{2} J_{1}=2
$$

among the differentiated invariants from which all others can be deduced by invariant differentiation.

\section{Recurrence Formulae.}

Since the basic differential invariants arising from invariantization of the jet coordinates form a complete system, any other differential invariant, e.g., those constructed by application of the invariant differential operators, can be locally written as a function thereof. The recurrence formulae, cf. $[\mathbf{2 2}, \mathbf{3 1}]$, connect the differentiated invariants and forms with their normalized counterparts. These formulae are fundamental, since they prescribe the structure of the algebra of (local) differential invariants, underly a full classification of generating differential invariants and their differential syzygies, as well as the structure of invariant variational problems and, indeed, the entire invariant variational bicomplex. As in the finite-dimensional version, the recurrence formulae are established, through just linear algebra and differentiation, using only the formulas for the prolonged infinitesimal generators and the cross-section. In particular, they do not require the explicit formulae for either the moving frame, or the Maurer-Cartan forms, or the normalized differential invariants and invariant forms, or even the invariant differential operators!

Let $\nu^{(\infty)}=\left(\rho^{(\infty)}\right)^{*} \mu^{(\infty)}$ denote the pulled-back Maurer-Cartan forms via the complete moving frame section $\rho^{(\infty)}$, with individual components

$$
\nu_{A}^{b}=\left(\rho^{(\infty)}\right)^{*}\left(\mu_{A}^{b}\right)=\sum_{i=1}^{p} S_{A, i}^{b} \omega^{i}+\sum_{\alpha, K} T_{A, \alpha}^{b, K} \vartheta_{K}^{\alpha}, \quad b=1, \ldots, m, \quad \# A \geq 0
$$

where the coefficients $S_{A, i}^{b}, T_{A, \alpha}^{b, K}$ will be called the Maurer-Cartan invariants. Their precise formulas will follow directly from the recurrence relations for the phantom differential 
invariants. In view of Theorem 3.2, the pulled-back Maurer-Cartan forms are subject to the linear relations

$$
L^{(n)}\left(H, I, \nu^{(n)}\right)=\iota\left[L^{(n)}\left(z, \zeta^{(n)}\right)\right]=0, \quad n \geq 0
$$

obtained by invariantizing the original linear determining equations (3.2), where we set $\iota\left(\zeta_{A}^{b}\right)=\nu_{A}^{b}$, and where $(H, I)=\iota(x, u)=\iota(z)$ are the zero ${ }^{\text {th }}$ order differential invariants in (4.6). In particular, if $\mathcal{G}$ acts transitively on $M$, then, since we are using a minimal order moving frame, $(H, I)$ are constant phantom invariants.

Given a locally defined vector field

$$
\mathbf{v}=\sum_{a=1}^{m} \zeta^{a}(z) \frac{\partial}{\partial z^{a}}=\sum_{i=1}^{p} \xi^{i}(x, u) \frac{\partial}{\partial x^{i}}+\sum_{\alpha=1}^{q} \varphi^{\alpha}(x, u) \frac{\partial}{\partial u^{\alpha}} \in \mathcal{X}(M),
$$

let

$$
\mathbf{v}^{(\infty)}=\sum_{i=1}^{p} \xi^{i}(x, u) \frac{\partial}{\partial x^{i}}+\sum_{\alpha=1}^{q} \sum_{k=\# J \geq 0} \widehat{\varphi}_{J}^{\alpha}\left(x, u^{(k)}\right) \frac{\partial}{\partial u_{J}^{\alpha}} \in \mathcal{X}\left(J^{\infty}(M, p)\right)
$$

denote its infinite prolongation. The coefficients are computed via the usual prolongation formula,

$$
\widehat{\varphi}_{J}^{\alpha}=D_{J} Q^{\alpha}+\sum_{i=1}^{p} u_{J, i}^{\alpha} \xi^{i}, \quad \text { where } \quad Q^{\alpha}=\varphi^{\alpha}-\sum_{i=1}^{p} u_{i}^{\alpha} \xi^{i}, \quad \alpha=1, \ldots, q
$$

are the components of the characteristic of $\mathbf{v}$; cf. $[\mathbf{5 1}, \mathbf{5 2}]$. Consequently, each prolonged vector field coefficient

$$
\widehat{\varphi}_{J}^{\alpha}=\Phi_{J}^{\alpha}\left(u^{(n)}, \zeta^{(n)}\right)
$$

is a certain universal linear combination of the vector field jet coordinates, whose coefficients are polynomials in the submanifold jet coordinates $u_{K}^{\beta}$ for $1 \leq \# K \leq n$. Let

$$
\eta^{i}=\iota\left(\xi^{i}\right)=\nu^{i}, \quad \widehat{\psi}_{J}^{\alpha}=\iota\left(\widehat{\varphi}_{J}^{\alpha}\right)=\Phi_{J}^{\alpha}\left(I^{(n)}, \nu^{(n)}\right),
$$

denote their invariantizations, which are certain linear combinations of the pulled-back Maurer-Cartan forms $\nu_{A}^{b}$, whose coefficients are polynomials in the normalized differential invariants $I_{K}^{\beta}$ for $1 \leq \# K \leq \# J$.

With all these in hand, the desired universal recurrence formula is as follows.

Theorem 5.1. If $\Omega$ is any differential form on $\mathrm{J}^{\infty}$, then

$$
d \iota(\Omega)=\iota\left[d \Omega+\mathbf{v}^{(\infty)}(\Omega)\right]
$$

where $\mathbf{v}^{(\infty)}(\Omega)$ denotes the Lie derivative of $\Omega$ with respect to the prolonged vector field (5.4), and we use (5.7) and its analogs for the partial derivatives of the prolonged vector field coefficients when invariantizing the result. 
Specializing $\Omega$ in (5.8) to be one of the coordinate functions $x^{i}, u_{J}^{\alpha}$ yields recurrence formulae for the normalized differential invariants (4.6),

$$
\begin{aligned}
& d H^{i}=\iota\left(d x^{i}+\xi^{i}\right)=\varpi^{i}+\eta^{i}, \\
& d I_{J}^{\alpha}=\iota\left(d u_{J}^{\alpha}+\widehat{\varphi}_{J}^{\alpha}\right)=\iota\left(\sum_{i=1}^{p} u_{J, i}^{\alpha} d x^{i}+\theta_{J}^{\alpha}+\widehat{\varphi}_{J}^{\alpha}\right)=\sum_{i=1}^{p} I_{J, i}^{\alpha} \varpi^{i}+\vartheta_{J}^{\alpha}+\widehat{\psi}_{J}^{\alpha},
\end{aligned}
$$

where, as in (5.7), each $\widehat{\psi}_{J}^{\alpha}$ is written in terms of the pulled-back Maurer-Cartan forms $\nu_{A}^{b}$, which are subject to the linear constraints (5.2). Each phantom differential invariant is, by definition, normalized to a constant value, and hence has zero differential. Consequently, the phantom recurrence formulae in (5.9) form a system of linear algebraic equations which can, as a result of the transversality of the cross-section, be uniquely solved for the pulledback Maurer-Cartan forms.

Theorem 5.2. If the pseudo-group acts locally freely on $\mathcal{V}^{n} \subset \mathrm{J}^{n}$, then the $n^{\text {th }}$ order phantom recurrence formulae can be uniquely solved to express the pulled-back Maurer-Cartan forms $\nu_{A}^{b}$ of order $\# A \leq n$ as invariant linear combinations of the invariant horizontal and contact one-forms $\varpi^{i}, \vartheta_{J}^{\alpha}$.

Substituting the resulting expressions (5.1) into the remaining, non-phantom recurrence formulae in (5.9) leads to a complete system of recurrence relations, for both the vertical and horizontal differentials of all the normalized differential invariants. In particular, equating the coefficients of the forms $\omega^{i}$ leads to individual recurrence formulae for the normalized differential invariants:

$$
\mathcal{D}_{i} H^{j}=\delta_{i}^{j}+R_{i}^{j}, \quad \mathcal{D}_{i} I_{J}^{\alpha}=I_{J, i}^{\alpha}+R_{J, i}^{\alpha},
$$

where $\delta_{i}^{j}$ is the Kronecker delta, and the correction terms $R_{i}^{j}, R_{J, i}^{\alpha}$ are certain invariant linear combinations of the Maurer-Cartan invariants $S_{A, i}^{b}$. One complication, which will be dealt with in the following section, is that the correction term $R_{J, i}^{\alpha}$ can have the same order as the initial differential invariant $I_{J, i}^{\alpha}$.

It is worth pointing out that, since the prolonged vector field coefficients $\widehat{\varphi}_{J}^{\alpha}$ are polynomials in the jet coordinates $u_{K}^{\beta}$ of order $\# K \geq 1$, their invariantizations are polynomial functions of the differential invariants $I_{K}^{\beta}$ for $\# K \geq 1$. Since the correction terms are constructed by solving a linear system for the invariantized Maurer-Cartan forms (5.1), the Maurer-Cartan invariants depend rationally on these differential invariants. Thus, in most cases (including the majority of applications), the resulting differential invariant algebra is endowed with an entirely rational algebraic recurrence structure.

Theorem 5.3. If $\mathcal{G}$ acts transitively on $M$, or, more generally, its infinitesimal generators depend polynomially on the coordinates $z=(x, u) \in M$, then the correction terms $R_{i}^{j}, R_{J, i}^{\alpha}$ in the recurrence formulas (5.9) are rational functions of the basic differential invariants. 


\section{The Symbol Modules.}

While the devil is in the details, the most important properties (Cartan characters, ellipticity, finite or infinite type, etc.) of a system of partial differential equations are fixed by the algebraic properties of its symbol module. For the action of a pseudo-group on submanifolds, there are, in fact, two interrelated submodules that prescribe the key structural features of the pseudo-group and its induced differential invariant algebra: the symbol module of its infinitesimal determining equations and the related prolonged symbol module governing its prolonged infinitesimal generators on the submanifold jet space. In particular, except for some low order complications, the generators of the differential invariant algebra and the associated differential syzygies can be identified with the algebraic generators and algebraic syzygies of an invariantized version of the prolonged symbol module. In this manner, constructive Gröbner basis techniques from commutative algebra can be applied to pin down the non-commutative differential algebraic structure of the pseudo-group's differential invariants.

To avoid technical complications, we will work in the analytic category. Let $\mathcal{G}$ be a pseudo-group, and let (3.2) be the formally integrable completion of its linearized determining equations. At each $z \in M$, we let $\left.\mathcal{I}\right|_{z}$ denote the symbol module of the determining equations, which, by involutivity, forms a submodule of the $\mathbb{R}[t]$ module

$$
\mathcal{T}=\left\{\eta(t, T)=\sum_{a=1}^{m} \eta_{a}(t) T^{a}\right\} \simeq \mathbb{R}[t] \otimes \mathbb{R}^{m}
$$

consisting of real polynomials in $t=\left(t_{1}, \ldots, t_{m}\right)$ and $T=\left(T^{1}, \ldots, T^{m}\right)$ that are linear in the $T$ 's. Assuming regularity, the symbol module's Hilbert polynomial, [16],

$$
H(n)=\sum_{i=0}^{d} b_{i}\left(\begin{array}{c}
n \\
d-i
\end{array}\right)
$$

where $b_{0}, b_{1}, \ldots, b_{d} \in \mathbb{Z}$, does not depend on $z \in M$. The integer $0 \leq d \leq m$ is the dimension, while $b=b_{0}$, its degree, is strictly positive unless $\left.\mathcal{I}\right|_{z}=\mathcal{T}$, in which case $\widetilde{H}(n) \equiv 0$ and the pseudo-group is purely discrete. Assuming solvability by the CartanKähler Theorem, $[\mathbf{8}, \mathbf{5 2}]$, the general solution to the determining equations, that is, the general pseudo-group transformation, can be written in terms of $b$ arbitrary functions of $d$ variables. In particular, the system is of finite type - and hence $\mathcal{G}$ is, in fact, a $b$ dimensional Lie group action - if and only if the symbol module has dimension $d=0$. See Seiler, $[\mathbf{6 4}, 65]$, for additional details.

The prolonged infinitesimal generators of the pseudo-group on the submanifold jet bundle have an analogous prolonged symbol module. Let

$$
\widehat{\mathcal{S}}=\left\{\widehat{\sigma}(s, S)=\sum_{\alpha=1}^{q} \widehat{\sigma}_{\alpha}(s) S^{\alpha}\right\} \simeq \mathbb{R}[s] \otimes \mathbb{R}^{q}
$$

be the $\mathbb{R}[s]$ module consisting of polynomials in $s=\left(s_{1}, \ldots, s_{p}\right), S=\left(S^{1}, \ldots, S^{q}\right)$, which are linear in the $S^{\prime}$ 's. At each submanifold 1-jet $z^{(1)}=\left(x, u^{(1)}\right)=\left(\ldots x^{i} \ldots u^{\alpha} \ldots u_{i}^{\alpha} \ldots\right) \in$ 
$\mathrm{J}^{1}(M, p)$, we define a linear map $\left.\boldsymbol{\beta}\right|_{z^{(1)}}: \mathbb{R}^{m} \times \mathbb{R}^{m} \rightarrow \mathbb{R}^{m}$ by the formulas

$$
\begin{array}{rlr}
s_{i} & =\beta_{i}\left(z^{(1)} ; t\right)=t_{i}+\sum_{\alpha=1}^{q} u_{i}^{\alpha} t_{p+\alpha}, & \\
S^{\alpha}=B^{\alpha}\left(z^{(1)} ; T\right)=T^{p+\alpha}-\sum_{i=1}^{p} u_{i}^{\alpha} T^{i}, \ldots, p, & &
\end{array}
$$

The induced pull-back map

$$
\begin{aligned}
\left(\left.\boldsymbol{\beta}\right|_{z^{(1)}}\right)^{*}\left[\widehat { \sigma } \left(s_{1}, \ldots,\right.\right. & \left.\left.s_{p}, S_{1}, \ldots, S_{q}\right)\right] \\
& =\widehat{\sigma}\left(\beta_{1}\left(z^{(1)} ; t\right), \ldots, \beta_{p}\left(z^{(1)} ; t\right), B^{1}\left(z^{(1)} ; T\right) \ldots, B^{q}\left(z^{(1)} ; T\right)\right)
\end{aligned}
$$

defines an injection $\left(\left.\boldsymbol{\beta}\right|_{z^{(1)}}\right)^{*}: \widehat{\mathcal{S}} \rightarrow \mathcal{T}$.

Definition 6.1. The prolonged symbol submodule at $\left.z^{(1)} \in \mathrm{J}^{1}\right|_{z}$ is the inverse image of the symbol module under this pull-back map:

$$
\left.\mathcal{J}\right|_{z^{(1)}}=\left(\left(\left.\boldsymbol{\beta}\right|_{z^{(1)}}\right)^{*}\right)^{-1}\left(\left.\mathcal{I}\right|_{z}\right)=\left\{\sigma(s, S)\left|\left(\left.\boldsymbol{\beta}\right|_{z^{(1)}}\right)^{*}(\sigma) \in \mathcal{I}\right|_{z}\right\} \subset \widehat{\mathcal{S}}
$$

It can be proved that, as long as $n>n^{\star}$, the module $\left.\mathcal{J}\right|_{z^{(1)}}$ coincides with the symbol module associated with the prolonged infinitesimal generators (5.4); see $[\mathbf{6 0}]$ for precise details.

To relate this construction to the differential invariant algebra, we need to invariantize the modules using our moving frame. In general, the invariantization of a prolonged symbol polynomial

$$
\sigma\left(x, u^{(1)} ; s, S\right)=\left.\sum_{\alpha, J} h_{\alpha}^{J}\left(x, u^{(1)}\right) s_{J} S^{\alpha} \in \mathcal{J}\right|_{z^{(1)}}, \quad \text { where } \quad z^{(1)}=\left(x, u^{(1)}\right),
$$

is given by

$$
\widetilde{\sigma}\left(H, I^{(1)} ; s, S\right)=\iota\left[\sigma\left(x, u^{(1)} ; s, S\right)\right]=\sum_{\alpha, J} h_{\alpha}^{J}\left(H, I^{(1)}\right) s_{J} S^{\alpha} .
$$

Let $\left.\widetilde{\mathcal{J}}\right|_{\left(H, I^{(1)}\right)}=\iota\left(\left.\mathcal{J}\right|_{z^{(1)}}\right)$ denote the resulting invariantized prolonged symbol submodule. We identify each parametrized symbol polynomial (6.7) with the differential invariant

$$
I_{\tilde{\sigma}}=\sum_{\alpha, J} h_{\alpha}^{J}\left(H, I^{(1)}\right) I_{J}^{\alpha}
$$

If $\mathcal{G}$ acts transitively on an open subset of $J^{1}$, then $\widetilde{\mathcal{J}}=\left.\widetilde{\mathcal{J}}\right|_{\left(H, I^{(1)}\right)}$ is a fixed module, independent of the submanifold jet coordinates, and (6.8) is a linear, constant coefficient combination of the normalized differential invariants.

The recurrence formulae for these differential invariants take the form

$$
\mathcal{D}_{i} I_{\tilde{\sigma}}=I_{s_{i} \tilde{\sigma}}+R_{\tilde{\sigma}, i}
$$


in which, as long as $n=\operatorname{deg} \widetilde{\sigma}>n^{\star}$, the leading term $I_{s_{i}} \tilde{\sigma}$ is a differential invariant of order $=n+1$, while, unlike in (5.10), the correction term $R_{\tilde{\sigma}, i}$ is of lower order $\leq n$. Iteration leads to the higher order recurrences

$$
\mathcal{D}_{J} I_{\tilde{\sigma}}=I_{s_{J} \tilde{\sigma}}+R_{\tilde{\sigma}, J},
$$

where $J=\left(j_{1}, \ldots, j_{k}\right)$ is an ordered multi-index of order $k$, and, assuming order $I_{\tilde{\sigma}}=$ $\operatorname{deg} \widetilde{\sigma}=n>n^{\star}$, the correction term $R_{\tilde{\sigma}, J}$ has order $<k+n=\operatorname{deg}\left[s_{J} \widetilde{\sigma}(s, S)\right]$.

With this in hand, we are able to state a Constructive Basis Theorem for the differential invariant algebra of an eventually locally freely acting pseudo-group.

Theorem 6.2. Let $\mathcal{G}$ be a Lie pseudo-group that acts locally freely on an open subset of the submanifold jet bundle at order $n^{\star}$. Then the following constitute a finite generating system for its algebra of local differential invariants:

(a) the differential invariants $I_{\nu}=I_{\sigma_{\nu}}$, where $\sigma_{1}, \ldots, \sigma_{l}$ form a Gröbner basis for the invariantized prolonged symbol submodule, and, possibly,

(b) a finite number of additional differential invariants of order $\leq n^{\star}$.

As noted above, the listed differential invariants do not typically form a minimal generating system, and the characterization of minimal generators remains a challenging open problem.

We are also able to exhibit a finite generating system of differential invariant syzygies - again not necessarily minimal. First, owing to the non-commutative nature of the the invariant differential operators, (4.9), we have the commutator syzygies

$$
\mathcal{D}_{J} I_{\tilde{\sigma}}-\mathcal{D}_{\widetilde{J}} I_{\tilde{\sigma}}=R_{\tilde{\sigma}, J}-R_{\tilde{\sigma}, \widetilde{J}} \equiv S_{J, \widetilde{J}, \tilde{\sigma}}, \quad \text { whenever } \quad \widetilde{J}=\pi(J)
$$

for some permutation $\pi$ of the multi-index $J$. Provided $\operatorname{deg} \widetilde{\sigma}>n^{\star}$, the right hand side $S_{J, \widetilde{J}, \tilde{\sigma}}$ is a differential invariant of lower order than those on the left hand side.

In addition, any commutative algebraic syzygy satisfied by polynomials in the prolonged symbol module $\left.\widetilde{\mathcal{J}}\right|_{\left(H, I^{(1)}\right)}$ provides an additional "essential" syzygy amongst the differentiated invariants. In detail, to each invariantly parametrized polynomial

$$
q\left(H, I^{(1)} ; s\right)=\sum_{J} q_{J}\left(H, I^{(1)}\right) s_{J} \in \mathbb{R}[s]
$$

we associate an invariant differential operator

$$
q\left(H, I^{(1)} ; \mathcal{D}\right)=\sum_{J} q_{J}\left(H, I^{(1)}\right) \mathcal{D}_{J} .
$$

Our convention is that the sums range over non-decreasing multi-indices $1 \leq j_{1} \leq j_{2} \leq$ $\cdots \leq j_{k} \leq p$, for $k=\# J$, and where, for specificity, we adopt the normal ordering when writing $\mathcal{D}_{J}=\mathcal{D}_{j_{1}} \mathcal{D}_{j_{2}} \cdots \mathcal{D}_{j_{k}}$. In view of $(6.10)$, whenever $\left.\widetilde{\sigma}\left(H, I^{(1)} ; s, S\right) \in \widetilde{\mathcal{J}}\right|_{\left(H, I^{(1)}\right)}$, we can write

$$
q\left(H, I^{(1)} ; \mathcal{D}\right) I_{\tilde{\sigma}\left(H, I^{(1)} ; s, S\right)}=I_{q\left(H, I^{(1)} ; s\right) \tilde{\sigma}\left(H, I^{(1)} ; s, S\right)}+R_{q, \tilde{\sigma}}
$$


where $R_{q, \tilde{\sigma}}$ has order $<\operatorname{deg} q+\operatorname{deg} \widetilde{\sigma}$. In particular, any algebraic syzygy

$$
\sum_{\nu=1}^{l} q_{\nu}\left(H, I^{(1)}, s\right) \sigma_{\nu}\left(H, I^{(1)} ; s, S\right)=0
$$

among the Gröbner basis polynomials of the invariantized prolonged symbol module induces a syzygy among the generating differential invariants:

$$
\sum_{\nu=1}^{l} q_{\nu}\left(H, I^{(1)}, \mathcal{D}\right) I_{\tilde{\sigma}_{\nu}}\left(H, I^{(1)} ; s, S\right)=R
$$

where $\operatorname{order} R<\max \left\{\operatorname{deg} q_{\nu}+\operatorname{deg} \widetilde{\sigma}_{\nu}\right\}$.

Theorem 6.3. Every differential syzygy among the generating differential invariants is a combination of the following:

(a) the syzygies among the differential invariants of order $\leq n^{\star}$,

(b) the commutator syzygies,

(c) syzygies coming from an algebraic syzygy among the Gröbner basis polynomials.

In this manner, we deduce a finite system of generating differential syzygies for the differential invariant algebra of our pseudo-group.

Further details, and applications of these results can be found in our papers listed in the references.

\section{References}

[1] Anderson, I.M., The Variational Bicomplex, Utah State Technical Report, 1989, http: //math.usu.edu/ fg_mp.

[2] Bazin, P.-L., and Boutin, M., Structure from motion: theoretical foundations of a novel approach using custom built invariants, SIAM J. Appl. Math. 64 (2004), 1156-1174.

[3] Berchenko, I.A., and Olver, P.J., Symmetries of polynomials, J. Symb. Comp. 29 (2000), 485-514.

[4] Bílă, N., Mansfield, E.L., and Clarkson, P.A., Symmetry group analysis of the shallow water and semi-geostrophic equations, Quart. J. Mech. Appl. Math., to appear.

[5] Bleecker, D., Gauge Theory and Variational Principles, Addison-Wesley Publ. Co., Reading, Mass., 1981.

[6] Boutin, M., Numerically invariant signature curves, Int. J. Computer Vision 40 (2000), 235-248.

[7] Boutin, M., On orbit dimensions under a simultaneous Lie group action on $n$ copies of a manifold, J. Lie Theory 12 (2002), 191-203. 
[8] Bryant, R.L., Chern, S.-S., Gardner, R.B., Goldschmidt, H.L., and Griffiths, P.A., Exterior Differential Systems, Math. Sci. Res. Inst. Publ., Vol. 18, Springer-Verlag, New York, 1991.

[9] Calabi, E., Olver, P.J., Shakiban, C., Tannenbaum, A., and Haker, S., Differential and numerically invariant signature curves applied to object recognition, Int. J. Computer Vision 26 (1998), 107-135.

[10] Cartan, É., La Méthode du Repère Mobile, la Théorie des Groupes Continus, et les Espaces Généralisés, Exposés de Géométrie, no. 5, Hermann, Paris, 1935.

[11] Cartan, É., Sur la structure des groupes infinis de transformations, in: Oeuvres Complètes, Part. II, Vol. 2, Gauthier-Villars, Paris, 1953, pp. 571-714.

[12] Cartan, É., La structure des groupes infinis, in: Oeuvres Complètes, part. II, vol. 2, Gauthier-Villars, Paris, 1953, pp. 1335-1384.

[13] Cheh, J., Olver, P.J., and Pohjanpelto, J., Maurer-Cartan equations for Lie symmetry pseudo-groups of differential equations, J. Math. Phys. 46 (2005), 023504 .

[14] Cheh, J., Olver, P.J., and Pohjanpelto, J., Algorithms for differential invariants of symmetry groups of differential equations, Found. Comput. Math., to appear.

[15] Chern, S.S., and Moser, J.K., Real hypersurfaces in complex manifolds, Acta Math. 133 (1974), 219-271; also Selected Papers, vol. 3, Springer-Verlag, New York, 1989, pp. 209-262.

[16] Cox, D., Little, J., and O'Shea, D., Ideals, Varieties, and Algorithms, 2nd ed., Springer-Verlag, New York, 1996.

[17] David, D., Kamran, N., Levi, D., and Winternitz, P., Subalgebras of loop algebras and symmetries of the Kadomtsev-Petviashivili equation, Phys. Rev. Lett. 55 (1985), 2111-2113.

[18] Di Francesco, P., Mathieu, P., and Sénéchal, D., Conformal Field Theory, Springer-Verlag, New York, 1997.

[19] Ehresmann, C., Introduction à la théorie des structures infinitésimales et des pseudo-groupes de Lie, in: Géometrie Différentielle, Colloq. Inter. du Centre Nat. de la Rech. Sci., Strasbourg, 1953, pp. 97-110.

[20] Fefferman, C., and Graham, C.R., Conformal invariants, in: Élie Cartan et les Mathématiques d'aujourd'hui, Astérisque, hors série, Soc. Math. France, Paris, 1985, pp. 95-116.

[21] Fels, M., and Olver, P.J., Moving coframes. I. A practical algorithm, Acta Appl. Math. 51 (1998), 161-213.

[22] Fels, M., and Olver, P.J., Moving coframes. II. Regularization and theoretical foundations, Acta Appl. Math. 55 (1999), 127-208.

[23] Fuchs, D.B., Gabrielov, A.M., and Gel'fand, I.M., The Gauss-Bonnet theorem and Atiyah-Patodi-Singer functionals for the characteristic classes of foliations, Topology 15 (1976), 165-188.

[24] Guggenheimer, H.W., Differential Geometry, McGraw-Hill, New York, 1963.

[25] Hubert, E.; The AIDA Maple package; http://www.inria.fr/cafe/Evelyne.Hubert/aida, 2006.. 
[26] Hubert, E., and Kogan, I.A., Rational invariants of an algebraic group action. Construction and rewriting, J. Symb. Comp., to appear.

[27] Hubert, E., and Kogan, I.A., Smooth and algebraic invariants of a group action. Local and global constructions, preprint, preprint, INRIA, 2005.

[28] Kamran, N., Contributions to the study of the equivalence problem of Elie Cartan and its applications to partial and ordinary differential equations, Mém. Cl. Sci. Acad. Roy. Belg. 45 (1989), Fac. 7.

[29] Kamran, N., and Robart, T., A manifold structure for analytic isotropy Lie pseudogroups of infinite type, J. Lie Theory 11 (2001), 57-80.

[30] Kim, P., Invariantization of Numerical Schemes for Differential Equations Using Moving Frames, Ph.D. Thesis, Ph.D. Thesis, University of Minnesota, Minneapolis, 2006.

[31] Kogan, I.A., and Olver, P.J., Invariant Euler-Lagrange equations and the invariant variational bicomplex, Acta Appl. Math. 76 (2003), 137-193.

[32] Kruglikov, B., and Lychagin, V., Invariants of pseudogroup actions: homological methods and finiteness theorem, preprint, arXiv: math.DG/0511711, 2005.

[33] Kumpera, A., Invariants différentiels d'un pseudogroupe de Lie, J. Diff. Geom. 10 (1975), 289-416.

[34] Kuranishi, M., On the local theory of continuous infinite pseudo groups I, Nagoya Math. J. 15 (1959), 225-260.

[35] Kuranishi, M., On the local theory of continuous infinite pseudo groups II, Nagoya Math. J. 19 (1961), 55-91.

[36] Lie, S., Die Grundlagen für die Theorie der unendlichen kontinuierlichen Transformationsgruppen, Leipzig. Ber. 43 (1891), 316-393; also Gesammelte Abhandlungen, Vol. 6, B.G. Teubner, Leipzig, 1927, pp. 300-364.

[37] Lisle, I.G., and Reid, G.J., Geometry and structure of Lie pseudogroups from infinitesimal defining systems, J. Symb. Comp. 26 (1998), 355-379.

[38] Lisle, I.G., and Reid, G.J., Cartan structure of infinite Lie pseudogroups, in: Geometric Approaches to Differential Equations, P.J. Vassiliou and I.G. Lisle, eds., Austral. Math. Soc. Lect. Ser., 15, Cambridge Univ. Press, Cambridge, 2000, pp. 116-145.

[39] Mackenzie, K., Lie Groupoids and Lie Algebroids in Differential Geometry, London Math. Soc. Lecture Notes, vol. 124, Cambridge University Press, Cambridge, 1987.

[40] Mansfield, E.L., Algorithms for symmetric differential systems, Found. Comput. Math. 1 (2001), 335-383.

[41] Marí Beffa, G., Relative and absolute differential invariants for conformal curves, $J$. Lie Theory 13 (2003), 213-245.

[42] Marí Beffa, G., Poisson geometry of differential invariants of curves in some nonsemisimple homogeneous spaces, Proc. Amer. Math. Soc. 134 (2006), 779-791.

[43] Marí Beffa, G., and Olver, P.J., Differential invariants for parametrized projective surfaces, Commun. Anal. Geom. 7 (1999), 807-839. 
[44] Martina, L., Sheftel, M.B., and Winternitz, P., Group foliation and non-invariant solutions of the heavenly equation, J. Phys. A 34 (2001), 9243-9263.

[45] McLachlan, R.I., and Quispel, G.R.W., What kinds of dynamics are there? Lie pseudogroups, dynamical systems and geometric integration, Nonlinearity 14 (2001), 1689-1705.

[46] Medolaghi, P., Classificazione delle equazioni alle derivate parziali del secondo ordine, che ammettono un gruppo infinito di trasformazioni puntuali, Ann. Mat. Pura Appl. 1 (3) (1898), 229-263.

[47] Morozov, O., Moving coframes and symmetries of differential equations, J. Phys. A 35 (2002), 2965-2977.

[48] Morozov, O.I., Structure of symmetry groups via Cartan's method: survey of four approaches, SIGMA: Symmetry Integrability Geom. Methods Appl. 1 (2005), paper 006.

[49] Muñoz, J., Muriel, F.J., and Rodríguez, J., On the finiteness of differential invariants, J. Math. Anal. Appl. 284 (2003), 266-282.

[50] Nutku, Y., and Sheftel, M.B., Differential invariants and group foliation for the complex Monge-Ampère equation, J. Phys. A 34 (2001), 137-156.

[51] Olver, P.J., Applications of Lie Groups to Differential Equations, Second Edition, Graduate Texts in Mathematics, vol. 107, Springer-Verlag, New York, 1993.

[52] Olver, P.J., Equivalence, Invariants, and Symmetry, Cambridge University Press, Cambridge, 1995.

[53] Olver, P.J., Classical Invariant Theory, London Math. Soc. Student Texts, vol. 44, Cambridge University Press, Cambridge, 1999.

[54] Olver, P.J., Joint invariant signatures, Found. Comput. Math. 1 (2001), 3-67.

[55] Olver, P.J., Geometric foundations of numerical algorithms and symmetry, Appl. Alg. Engin. Commun. Comput. 11 (2001), 417-436.

[56] Olver, P.J., Generating differential invariants, preprint, University of Minnesota, 2006.

[57] Olver, P.J., and Pohjanpelto, J., Regularity of pseudogroup orbits, in: Symmetry and Perturbation Theory, G. Gaeta, B. Prinari, S. Rauch-Wojciechowski, S. Terracini, eds., World Scientific, Singapore, 2005, pp. 244-254.

[58] Olver, P.J., and Pohjanpelto, J., Maurer-Cartan forms and the structure of Lie pseudo-groups, Selecta Math. 11 (2005), 99-126.

[59] Olver, P.J., and Pohjanpelto, J., Moving frames for Lie pseudo-groups, Canadian J. Math., to appear.

[60] Olver, P.J., and Pohjanpelto, J., On the algebra of differential invariants of a Lie pseudo-group, preprint, University of Minnesota, 2006.

[61] Ovsiannikov, L.V., Group Analysis of Differential Equations, Academic Press, New York, 1982.

[62] Pommaret, J.F., Systems of Partial Differential Equations and Lie Pseudogroups, Gordon and Breach, New York, 1978.

[63] Robart, T., and Kamran, N., Sur la théorie locale des pseudogroupes de transformations continus infinis I, Math. Ann. 308 (1997), 593-613. 
[64] Seiler, W., On the arbitrariness of the solution to a general partial differential equation, J. Math. Phys. 35 (1994), 486-498.

[65] Seiler, W., Involution, in preparation.

[66] Serra, J., Image Analysis and Mathematical Morphology, Academic Press, London 1982.

[67] Singer, I.M., and Sternberg, S., The infinite groups of Lie and Cartan. Part I (the transitive groups), J. Analyse Math. 15 (1965), 1-114.

[68] Stormark, O., Lie's Structural Approach to PDE Systems, Cambridge University Press, Cambridge, 2000.

[69] Tresse, A., Sur les invariants différentiels des groupes continus de transformations, Acta Math. 18 (1894), 1-88.

[70] Tsujishita, T., On variational bicomplexes associated to differential equations, Osaka J. Math. 19 (1982), 311-363.

[71] Vessiot, E., Sur l'intégration des systèmes différentiels qui admettent des groupes continues de transformations, Acta. Math. 28 (1904), 307-349.

[72] Welk, M., Kim, P., and Olver, P.J., Numerical invariantization for morphological PDE schemes, preprint, University of Minnesota, 2006. 